\title{
Adsorption of Molecular Gases on Silver/Carbon Nanotube Composites at Low Temperatures and Low Pressures
}

\author{
M. Barberio, ${ }^{1,2}$ P. Barone, ${ }^{1,2}$ F. Stranges, ${ }^{1,2}$ R. A. Romano, ${ }^{1}$ F. Xu, ${ }^{1}$ and A. Bonanno ${ }^{1}$ \\ ${ }^{1}$ Dipartimento di Fisica, Università della Calabria, Via Bucci 31 C, 87036 Rende, Italy \\ ${ }^{2}$ Dipartimento di Biologia, Ecologia e Scienze della Terra, Università della Calabria, Via Bucci 4B, 87036 Rende, Italy \\ Correspondence should be addressed to M. Barberio; marianna.barberio@fis.unical.it
}

Received 20 May 2013; Revised 17 October 2013; Accepted 21 October 2013; Published 9 January 2014

Academic Editor: Yang Xu

Copyright (c) 2014 M. Barberio et al. This is an open access article distributed under the Creative Commons Attribution License, which permits unrestricted use, distribution, and reproduction in any medium, provided the original work is properly cited.

\begin{abstract}
We present an experimental study adsorption of molecular gases $\left(\mathrm{N}_{2}, \mathrm{H}_{2}, \mathrm{O}_{2}, \mathrm{CH}_{4}, \mathrm{C}_{2} \mathrm{H}_{4}\right.$, and $\left.\mathrm{C}_{2} \mathrm{H}_{6}\right)$ on multiwalled carbon nanotubes (MWCNTs) and MWCNT doped with Ag at low temperatures ( $35 \mathrm{~K})$ and pressures $\left(10^{-6}\right.$ Torr) using the temperature programmed desorption technique. Our results show that the desorption kinetics is of the first order; furthermore comparative measurements indicate that $\mathrm{Ag} / \mathrm{MWCNTs}$ have an adsorption capacity higher than that of a pure sample suggesting that these composites are good candidates as gas cryosorbers for applications in cryopumps or sensor of latest generation.
\end{abstract}

\section{Introduction}

In last decades, carbon nanotubes have been intensively studied because of their importance as building blocks in nanotechnology [1-3]. In particular, gas adsorption on carbon nanotubes and nanotubes bundles is an important issue for both fundamental research in nanotubes and their technical applications [4-6]. Considerable experimental and theoretical efforts have been dedicated to hydrogen storage in nanotube based materials [4-6], but the changes in electronic properties of carbon nanotubes upon exposure to gases as $\mathrm{O}_{2}, \mathrm{NO}_{2}, \mathrm{NH}_{3}$, or $\mathrm{CH}_{4}$ are also object of several studies for application to sensor.

Recently we proposed to use multiwalled carbon nanotubes (MWCNTs) as hydrogen cryosorber in cryopumps and accelerators of latest generation [7]. We have demonstrated that MWCNT has, in conditions of very low temperature and pressure, an adsorption capacity about two orders of magnitude higher than that of activated carbon (charcoal) [7].

A means to achieve a high uptake of gas on a surface is to vary the porosity and the specific area of the adsorption surfaces, so that trapping and condensation of molecules inside the pores increase. Carbon nanotubes are good candidates for gas storage because of their high specific surface area and large volume of pores. Therefore it can be interesting to study the doping effects of nanoparticles on specific surface of carbon nanotubes. In fact, metallic nanoparticles possess a large surface area which makes them highly reactive in interactions with gas molecules. So, the insertion of metallic nanoparticles in nanotube networks can lead to the formation of composites with very great surface area. Recently we introduced a simple method to obtain composites based on carbon nanotubes doped with Ag nanoparticles [8]. We obtained that the Ag doping produces strong changes in electronic and optical properties of carbon nanotubes at very low nanoparticle concentration [8]. In this work we investigate the possibility to use these new materials as gas cryosorbers and demonstrate that the Ag doping improves the adsorption without changing the physical properties of chemical bonds between gas and surfaces. We report data of adsorption for different gases $\left(\mathrm{N}_{2}, \mathrm{H}_{2}, \mathrm{O}_{2}, \mathrm{CH}_{4}, \mathrm{C}_{2} \mathrm{H}_{4}\right.$, and $\mathrm{C}_{2} \mathrm{H}_{6}$ ) at very low temperature and pressure and compare the results with those obtained from pure MWCNT and pure copper surfaces. 


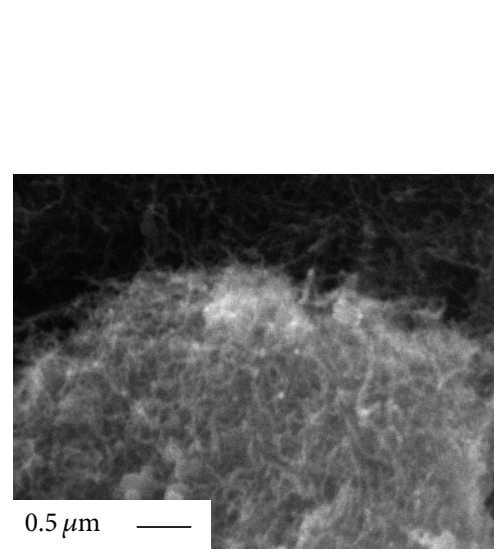

(a)

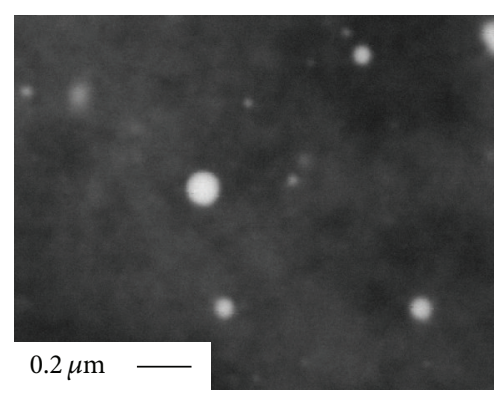

(b)

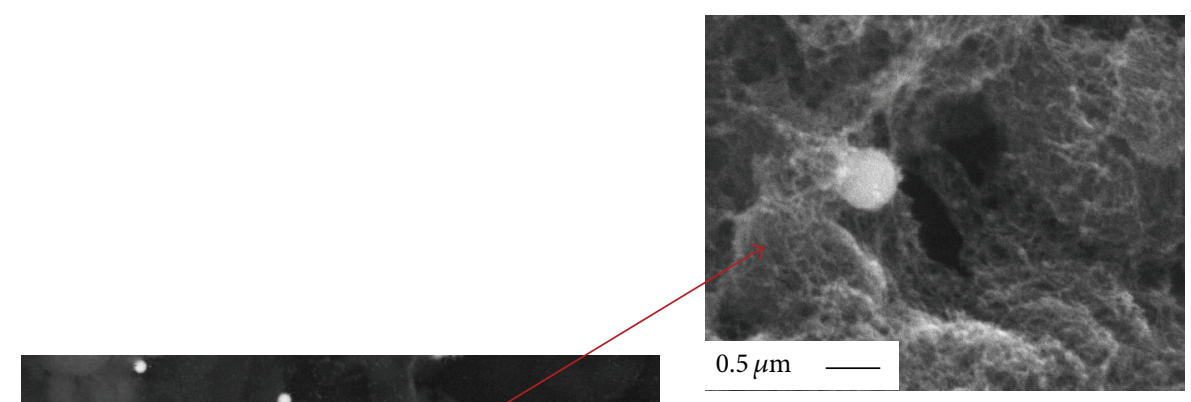

(d)

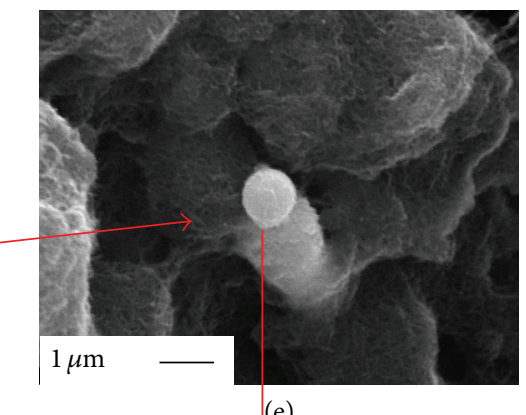

$(\mathrm{e})$

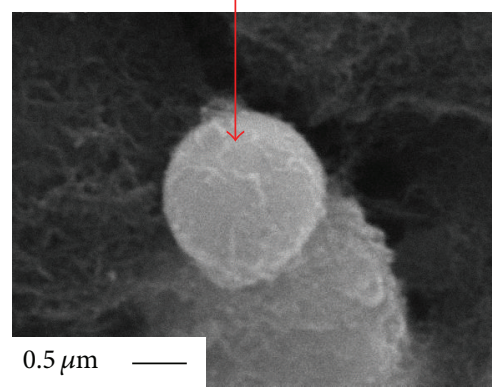

(f)

FIGURE 1: SEM images of Ag/CNT sample: (a) BSE image and (b) corresponding SE image of sample produced by process A. (c) BSE and (d) SE details on silver microparticles; (e-f) SE images of a silver microparticle completely covered by CNT.

The composites were produced by mixing commercial MWCNT and Ag nanoparticles obtained by laser ablation (LA). The produced nanoparticles were monitored by optical absorption measurements and SEM images, while the composites were characterized by X-ray photoelectron spectroscopy (XPS) and SEM images. The gas adsorption was studied by temperature programmed desorption (TPD) technique.

\section{Experiments}

2.1. Materials. The MWCNT powder used in this study was purchased from "Nanostructured \& Amorphous Materials, Inc.", with a nominal purity greater than $95 \%$. The samples are characterized (SEM analysis in Figure 1(a)) by the presence of CNT bundles with an average diameter of $30 \mathrm{~nm}$ and 5$20 \mu \mathrm{m}$ in length.

The powder of silver microparticles is obtained by laser ablation process on silver bar (nominal purity of $99.99 \%$ purchased from Goodfellow) which leads to formation of spherical and spheroid particles with dimensions of about
600-500 nm. Laser ablation was performed on silver bar placed vertically in a glass vessel filled with a $20 \mathrm{~mL}$ of acetone. We carried out the LA process with the first harmonic (1064 nm) of a Quanta-Giant series $710 \mathrm{Nd}$ :YAG laser operating at $10 \mathrm{~Hz}$ (laser power is fixed at $600 \mathrm{~mJ} /$ pulse). Upon irradiation of the laser beam, the solution, initially transparent, gradually turns brownish-yellow indicating the formation of Ag nanoparticles in solution [9]. After the LA, the solution is sonicated to prevent the self-aggregation of microparticles suspended in acetone and the formation of macroclusters. SEM images of Ag particles obtained by the analysis in backscattered electron mode (BSE) (Figure 1(b)) show the formation of spherical silver particles with dimensions ranging from 100 to $500 \mathrm{~nm}$. Shape and dimensions of silver nanoparticles were monitored during LA process by measuring the optical absorbance of the solution [9]. The process was stopped when the optimal conditions of shape and dimensions were reached.

Silver/carbon nanotubes composite structures were prepared using a simple chemical mixing method as indicated in Yao et al. $[10,11]$ and improved in several of our works 
to obtain CNT based heterostructures [10, and references therein]. This method is a sequence of mixing, evaporation, and drying processes. In the first step, carbon nanotubes and the acetone solution containing silver particles are dispersed in dichlorobenzene $\left(\mathrm{C}_{6} \mathrm{H}_{4} \mathrm{Cl}_{2}\right)$ as solvent and then sonicated for $1 \mathrm{~h}$. After sonication the suspension containing CNTs and silver is deposited on a copper plate $(1 \mathrm{~cm} \times 2 \mathrm{~cm})$, previously sonicated in acetone, and then heated at $180^{\circ} \mathrm{C}$ in air on a hot plate for 15 minutes to allow solvent evaporation and a fairly accurate cleanness of carbon nanotubes. In effect the carbon nanotubes cleanness, as demonstrated in our previous works [10], is effective only after some heating cycles at high temperature in ultra-high vacuum systems. Using one heating cycle in air at low temperature allows removing only the amorphous carbon, but not the oxygen. The BSE-SEM images of the composites show spherical silver microparticles with dimensions of about $100-500 \mathrm{~nm}$ randomly dispersed on MWCNT bulk (Figure 1(c)). Images at higher zoom level obtained in secondary electron mode (SE-SEM) indicate that each nanoparticle is inserted in MWCNT bulk and wrapped by carbon nanotubes (Figures 1(d), 1(e), and 1(f)).

2.2. Methods. The transmitted spectrum was obtained by a Horiba VS-140 VIS-NIR spectrometer working in the $350-1200 \mathrm{~nm}$ range. A white led has been used as light source to enlighten the solution Ag-acetone (the vessel glass surface containing the solution is completely transparent to white light). Transmittance $(t(\lambda))$ and absorbance $(a(\lambda))$ as function of wavelength are obtained with the Beer-Lambert law:

$$
t(\lambda)=\frac{I_{t}(\lambda)}{I_{s}(\lambda)}
$$

$a(\lambda)=-\ln (t(\lambda))$, where $I_{t}(\lambda)$ is the transmitted light and $I_{s}(\lambda)$ is the source light.

The transmitted spectrum was monitored during the whole laser ablation process, taking spectra at regular intervals of 5 seconds. At time $t$, the absorbance intensity $A(t)$ of each spectral transition was obtained by numerical integration over $\lambda$, assuming a Gaussian distribution of the spectral bands (Figure 2).

XPS measurements were conducted in a UHV chamber equipped for standard surface analysis with a pressure in the range of $10^{-9}$ Torr. Nonmonochromatic Mg-K $\alpha$ X-ray $(h v=1253.64 \mathrm{eV})$ was used as excitation source. The XPS spectra were calibrated with the $\mathrm{C}$ 1s peak of a pure carbon sample (energy position $284.6 \mathrm{eV}$ ). All XPS spectra have been corrected for the analyzer transmission factor and background subtraction obtained by using the straight line subtraction mode. Moreover the XPS data were fitted assuming a Gaussian distribution.

The thermal programmed desorption (TPD) experiments were conducted in a UHV chamber (base pressure in the low $10^{-9}$ Torr range) evacuated with a dry turbo pump (more information about experimental setup is in $[7,12,13])$. The gas $\left(\mathrm{N}_{2}, \mathrm{H}_{2}, \mathrm{O}_{2}, \mathrm{CH}_{4}, \mathrm{C}_{2} \mathrm{H}_{4}\right.$, and $\mathrm{C}_{2} \mathrm{H}_{6}$ with purity of $\left.99.999 \%\right)$ was introduced in the chamber through a precision leak valve while keeping the sample at an adsorption temperature of

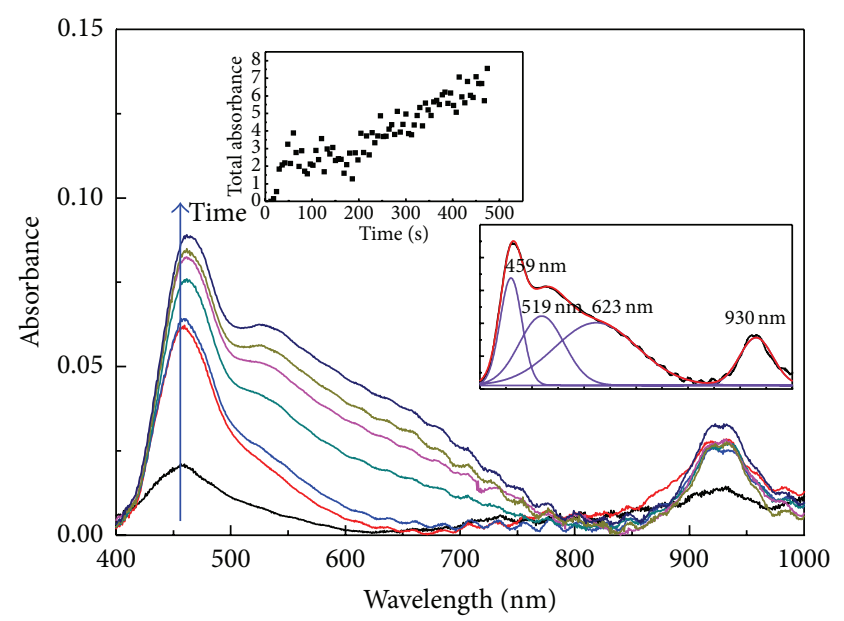

Figure 2: Absorbance spectra taken at regular interval of 50 seconds. Right inset: Gaussian analysis of the spectrum acquired after $500 \mathrm{~s}$ as an example of data treatment. Left inset: total optical absorbance versus LA irradiation time.

$T_{\mathrm{ad}}=35 \mathrm{~K}$ and at a fixed pressure of $1 \times 10^{-6} \mathrm{Torr}$ (measured at room temperature and corrected for the ion gauge sensitivity factor), for a chosen time interval. The samples $(3 \mathrm{mg}$ for each sample) were deposited directly onto the copper head $\left(6 \mathrm{~cm}^{2}\right)$ of a cold finger with closed He cycling and dried. A quite uniform thin film was obtained and its adhesion to the copper surface remained stable during all the measurements. The sample temperature was monitored with an $\mathrm{Au}=$ $0: 7 \% \mathrm{Fe}$-chromel thermocouple. Thermal desorption was achieved by heating the head of the cold finger with a linear ramp $(10 \mathrm{~K} / \mathrm{min})$ and the desorbed gas was measured with a quadrupole mass analyzer. No appreciable gas contamination signals were detected neither in the adsorption nor in the desorption processes. Complete reversibility of charging and discharging cycles was routinely verified and a total reproducibility was observed. The desorption rate was obtained by multiplying the detected gas partial pressure (the integral intensity of a quadrupole mass spectrum centered at specific gas atomic mass and composed of some 50 data points) by the vacuum system pumping speed ( $1 / \mathrm{s}$ technical data). The total amount of desorbed gas then could be evaluated from the integration of the desorption rate-time curves with an estimated error of about $5 \%$.

\section{Results and Discussion}

Conventionally metal nanoparticles are characterized by their extinction spectra (absorption and/or scattering spectra) [9]. The peaks observed in absorption or in scattering spectra of particles, during the light excitation, are due to resonant wavelengths of plasmon eigenmodes (called dipole plasmon resonance) of the particles as demonstrated in [9]. Secondary oscillations, called quadrupole plasmon resonances, appear at lower energy for larger particles [9, and references therein]. The plasmon oscillation frequency is determined by four factors: the density of electrons, the 


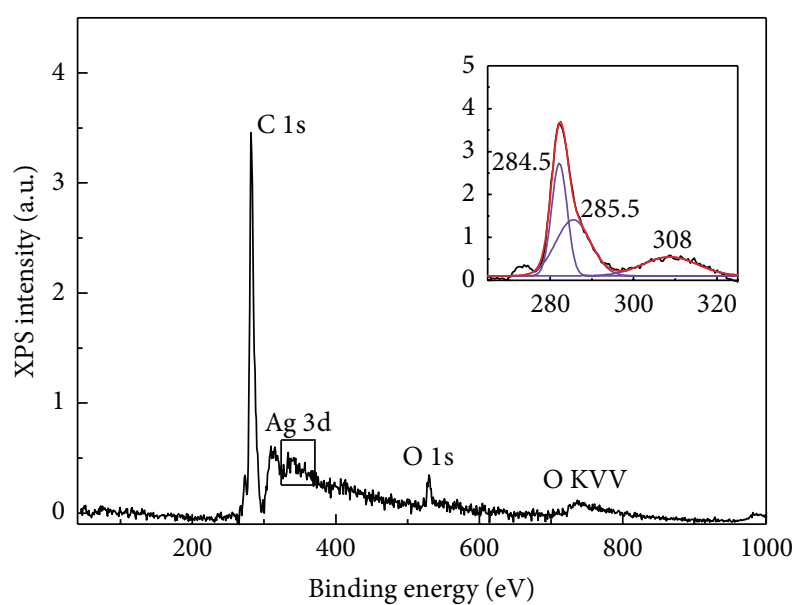

FIGURE 3: XPS survey spectrum taken from Ag/CNT composites. Inset: Gaussian analysis of C 1s line.

effective electron masses, and size and shape of charge distribution (i.e., by shape and size of nanoparticles) [9].

We collected, for 500 seconds, the absorbance spectrum of acetone-nanoparticles solution during LA at regular time intervals of 5 seconds, monitoring the changes in its intensity and line shape. Figure 2 shows, as example of typical measured, the absorbance spectra at intervals of 50 seconds. We observe that during the first 50 seconds the spectra exhibit two bands centered at about $460 \mathrm{~nm}$ and $930 \mathrm{~nm}$ with a full width at half maximum (FWHM) of $30 \mathrm{~nm}$ and $60 \mathrm{~nm}$, respectively. After the first 100 seconds, a third band appears at about $520 \mathrm{~nm}$ while after $200 \mathrm{~s}$ a band at $620 \mathrm{~nm}$ emerges (see the right inset in Figure 2 for Gaussian analysis of spectrum taken at $500 \mathrm{~s}$ ). All the four bands can be associated with oscillation of surface plasmon in spherical or spheroid nanoparticles. Kelly et al. in their theoretical work [9] calculated the plasmon absorption wavelength for both spherical and spheroid nanoparticles as function of particles dimensions. They demonstrated that spherical particles present two absorption bands centered at about $350 \mathrm{~nm}$ (dipole resonance) and at 440-460 nm (quadrupole resonance) for particles with dimensions of $60 \mathrm{~nm}$. These positions change strongly with particles dimensions and shape. If the sphere diameter increases, the bands are redshifted and can reach the NIR region if the particles are large spheroids.

So, our absorption spectra (with three resonance bands) can be interpreted as superposition of signals from particles of different sizes in solution. In the first 50 seconds, LA produces spherical particles which generate the resonance at $460 \mathrm{~nm}$. The dimensions of these particles, from the comparison with numerical data in [9], range between 60 and $70 \mathrm{~nm}$. Moreover in the first phases a little amount of spheroid particles is also present (resonance at $930 \mathrm{~nm}$ ). After the first 50 seconds the particle concentration in solutions increases and several nanoparticles aggregate to form spherical particles with larger dimensions causing the 520 and 620 band appearance. As the LA time increases, several particles aggregate forming larger spheres, while spheroid particles increase their concentration without changing size. SEM images in Figure 1(b) show spherical particles obtained after $500 \mathrm{~s}$ of LA, confirming the interpretation of our absorption spectra. The particle deposited after a LA time of $500 \mathrm{~s}$ is mainly spherical with a diameter of about $100-500 \mathrm{~nm}$. In our deposited samples only a little amount of spheroid particles is observed as evidenced by the small increases of the $930 \mathrm{~nm}$ band. Moreover the total optical absorbance obtained as time integration of absorbance spectra (see left inset in Figure 2) increases with LA irradiation time, indicating the increases of silver nanoparticle amount in solution.

XPS survey spectrum in Figure 3 shows the presence in our Ag/CNT composites of carbon, oxygen and silver without signs of impurities caused by the growth processes. The $\mathrm{C}$ 1s line (inset in Figure 3) is asymmetric and cannot be reproduced by one single Gaussian. Our fit indicates the presence of three well-distinguished peaks at 284.3 (main structure of C 1s), $285.6 \mathrm{eV}$ (C-O chemical bonds), and $308 \mathrm{eV}$ that are typical of carbon structures. In particular the broad structure at $302 \mathrm{eV}$ is due to plasma loss with both $\pi$ and $\sigma$ characters [14]. The $\mathrm{O} 1 \mathrm{~s}$ lines present a main structure at roughly $532 \mathrm{eV}$; this structure is often assigned to oxygen bonded to $\mathrm{C}$ indicating a single $\mathrm{C}-\mathrm{O}$ bonding [14] The presence of $\mathrm{C}-\mathrm{O}$ chemical bonds in the spectra is due to oxygen adsorbed in carbon nanotubes before the mixing with silver particles. The Ag $3 \mathrm{~d}$ lines are not structured and do not present a shift with respect to lines taken from a pure silver surface. This excludes the formation of chemical bonds between CNT or oxygen and Ag.

The element percentage in composites, calculated by XPS data, indicates a very little presence of silver on the surface (about $0.1 \%$ ). Besides this low doping, microporosity measurements indicate a strong increases of specific surface area of $\mathrm{Ag} / \mathrm{CNT}$ with respect to pure MWCNT. In fact the specific surface for pure MWCNT is of $174 \mathrm{~m}^{2} / \mathrm{g}$ while for $\mathrm{Ag} / \mathrm{CNT}$ composites are $2394 \mathrm{~m}^{2} / \mathrm{g}$. Moreover the sample roughness, measured under AFM condition, increases of about $16 \%$ in sample with silver nanoparticles $(70.8 \mathrm{~nm}$ for pure MWCNT and $83.3 \mathrm{~nm}$ for Ag/CNT composites).

So, XPS and microporosity results indicate that Ag doping of CNT is a simple physisorption without any signs of chemical bonds; the only effect of doping is changing the porosity of surface. These results are also confirmed by the increases in desorption for all tested gases on $\mathrm{Ag} / \mathrm{CNT}$ composites.

In Figure 4(a) we illustrate some representative TPD traces for different gases at dose $1000 \mathrm{~L}$ (the adsorption temperature is for all gases fixed at $T_{\text {ad }}=35 \mathrm{~K}$ ). The position of the desorption temperature changes with gas species indicating a different adsorption energy for different gases; in fact, it is known that the binding energy of a molecule on a surface varies with the adsorbate/surface combination [15].

Moreover Figure 4(b) shows, as example for all gas, the changes in adsorption, varying the exposure dose $(1 \mathrm{~L}=$ $10^{-6}$ Torr sec and the sample heating rate was fixed at $1 \mathrm{~K}=$ min) of $\mathrm{C}_{2} \mathrm{H}_{6}$; the behavior of all gases on both pure MWCNT and $\mathrm{Ag} / \mathrm{MWCNT}$ is identical.

These TPD traces are quite similar to each other and the temperature position of the spectrum maxima remains unchanged as the gas dose adsorption increases, clearly 


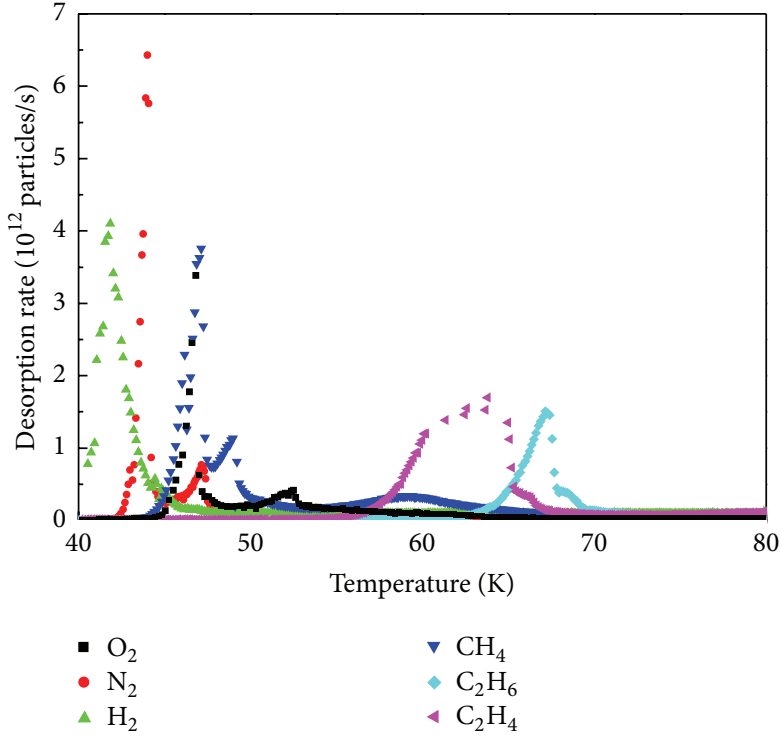

(a)

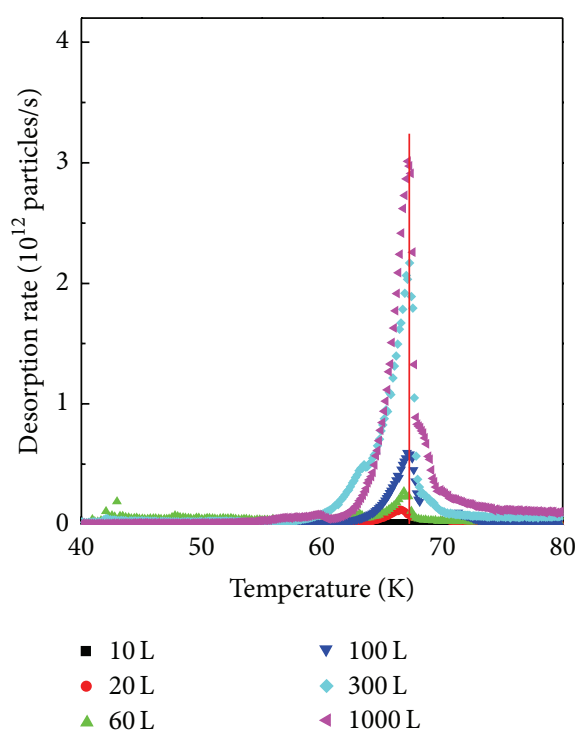

(b)

FIGURE 4: Temperature programmed desorption traces of molecular gases adsorbed on Ag/MWCNT composites at a dose of $1000 \mathrm{~L}$ (a). TPD traces of adsorbed $\mathrm{C}_{2} \mathrm{H}_{6}$ at different doses as example for all gases (b).

indicating a first-order desorption mechanism. This indicates interaction only between gas molecules and substrates, without gas-gas interplay on the sample surface. The position of the maximum in the desorption spectrum, indicated in Table 1, is identical (in the limit of experimental error), for each gas, on both samples and on cold head copper surface. This indicates that the absorption energy is unchanged for different materials; consequently in all cases the gases are physisorbed on surfaces without formation of chemical bonds with the substrate. Moreover the desorption temperature, as demonstrated in our previous work [7], is associated with the absorption energies which are less than those required for chemisorption processes $(>50 \mathrm{~kJ} / \mathrm{mol})$.

The only exception is the behavior of oxygen that (as visible from data in Table 1) shows a desorption temperature of about $43 \mathrm{~K}$ on all surface and a second desorption at about $50 \mathrm{~K}$ on $\mathrm{Ag} / \mathrm{MWCNT}$ composites. We attribute this behavior to great interaction between silver nanoparticles and oxygen. In our recent work [8] we demonstrate that silver nanoparticles exposed to atmospheric oxygen interact with oxygen to form silver oxide also in this case the exposure to $\mathrm{O}_{2}$ leads, as well as the oxygen absorption on surface, to the formation of silver oxide on Ag/MWCNT (as indicated by the Ag $3 \mathrm{~d}$ XPS lines). So, the physisorbed oxygen is desorbed at $45 \mathrm{~K}$ (as on MWCNT or copper) while the chemisorbed oxygen is desorbed at higher temperature of $50 \mathrm{~K}$.

The total molecular gas content, as determined from integration of the desorbed gas TPD trace curves, is plotted, for all gases and for all analyzed surfaces, in Figure 5 as a function of gas doses. It is interesting to note that it increases with doses for all surfaces, and for many of investigated gases the adsorption on Ag/MWCNT composites is greater than that on pure MWCNT, confirming the increases on
TABLE 1: Desorption temperatures (K).

\begin{tabular}{lcccccc}
\hline & $\mathrm{C}_{2} \mathrm{H}_{4}$ & $\mathrm{C}_{2} \mathrm{H}_{6}$ & $\mathrm{CH}_{4}$ & $\mathrm{H}_{2}$ & $\mathrm{~N}_{2}$ & $\mathrm{O}_{2}$ \\
\hline Copper & 65.07 & 70.22 & 48.5 & 42.7 & 44.4 & 45.27 \\
MWCNT & 65.25 & 69.39 & 47.58 & 41.7 & 43.9 & 43.1 \\
Ag/MWCNT & 64.41 & 70.4 & 48.67 & 41.6 & 44 & $43 / 50$ \\
\hline
\end{tabular}

specific area by doping with Ag nanoparticles. Only for $\mathrm{H}_{2}$ and $\mathrm{CH}_{4}$ the adsorption is comparable in pure and doped samples. Experiments on the absorption temperature of both these gases on carbon nanotubes and copper surfaces [7, 13] demonstrated that the adsorption is maximum at about 10$12 \mathrm{~K}$ and decreases strongly with temperature. So we can hypothesize that the adsorption temperature in this work is too high for $\mathrm{H}_{2}$ and $\mathrm{CH}_{4}$ adsorption also for Ag doped CNT.

\section{Conclusions}

In this work we present a study of gas adsorption on Ag doped MWCNT. The Ag/MWCNT composites show an increase in specific area of about 15 times as compared with a pure MWCNT surface, even with a very low Ag doping (0.1\%). The adsorption rate, which increases for many investigated gases $\left(\mathrm{N}_{2}, \mathrm{O}_{2}, \mathrm{C}_{2} \mathrm{H}_{4}\right.$, and $\left.\mathrm{C}_{2} \mathrm{H}_{6}\right)$, shows in all cases a firstorder adsorption process. The desorption temperatures, and then the absorption energies, are unchanged between pure MWCNT and Ag/MWCNT composites, clearly indicating that the absorption is on both samples a physisorption process without formation of chemical bonds between gases and substrate. The only exception is $\mathrm{O}_{2}$, since the silver nanoparticles are most reactive to oxygen and the $\mathrm{O}_{2}$ absorption leads to the formation of silver oxide on composite 


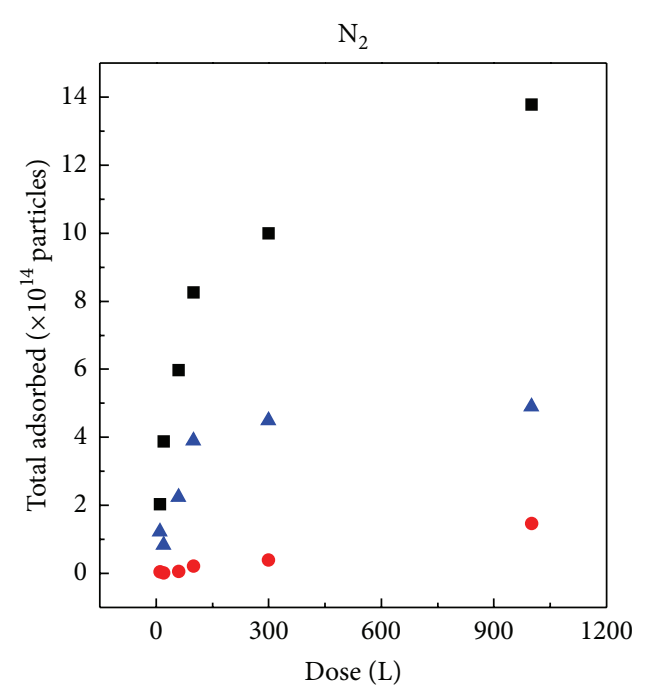

(a)

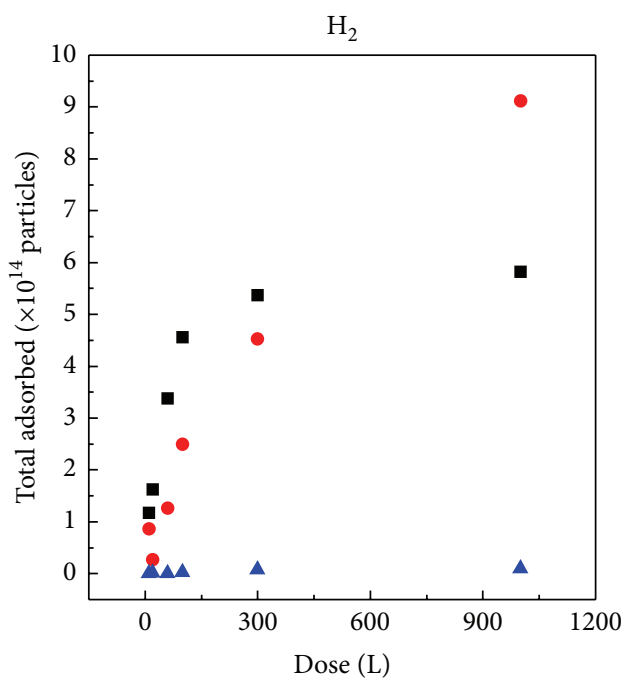

(c)

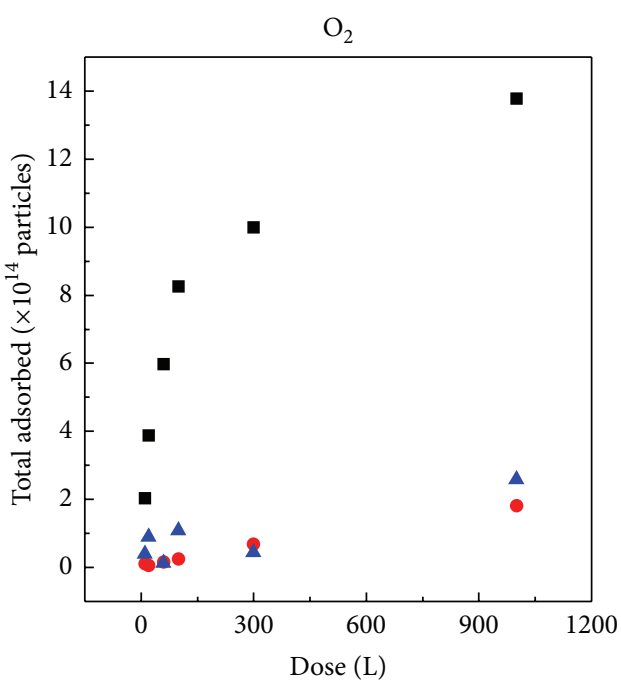

MWNT/Ag

- MWNT

$\Delta \mathrm{Cu}$

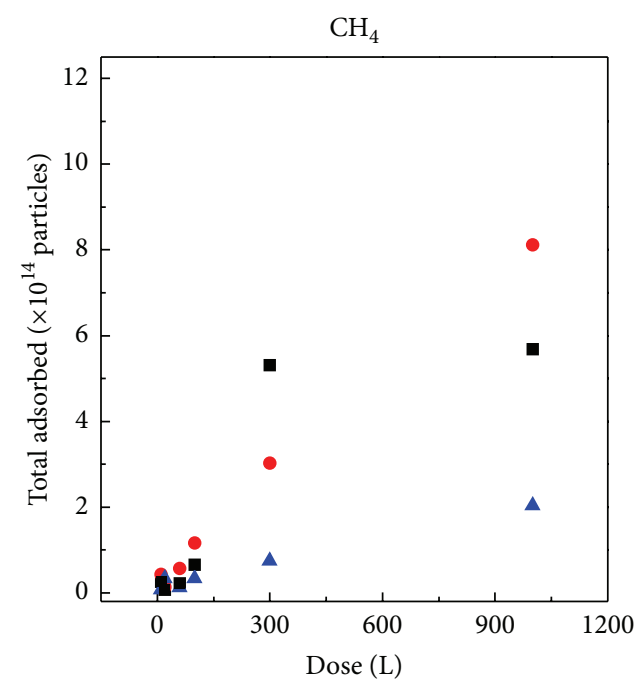

(b)

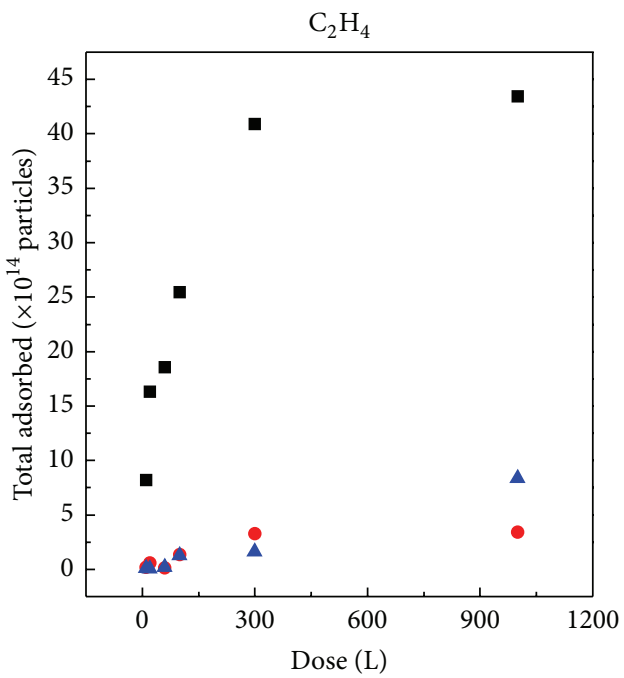

(d)

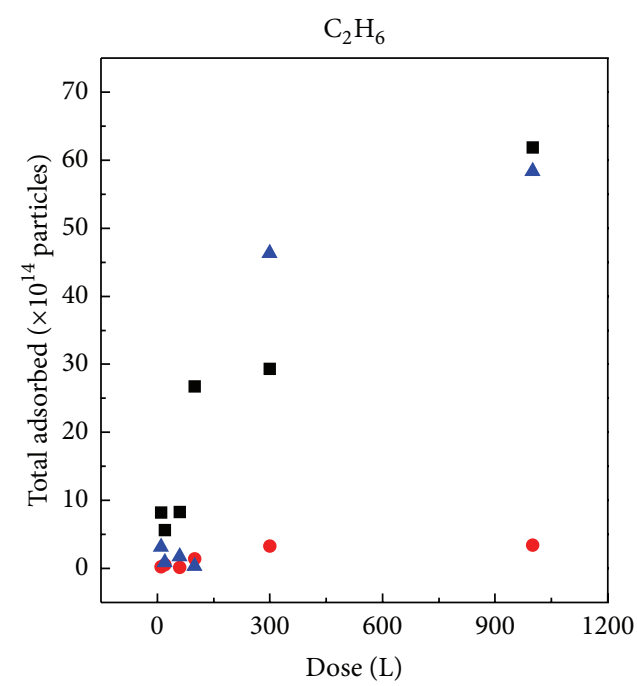

MWNT/Ag

MWNT

$\Delta \mathrm{Cu}$

(e)

(f)

FIgURE 5: Molecular gases adsorbed on MWCNT, Ag/MWCNT, and copper cold head surface as a function of gases dose. 
surfaces and to the appearance of a second peak, at higher energy, in the TPD spectrum.

\section{Conflict of Interests}

The authors declare that there is no conflict of interests regarding the publication of this paper.

\section{References}

[1] E. Fortunati, F. D’Angelo, S. Martino, A. Orlacchio, J. M. Kenny, and I. Armentano, "Carbon nanotubes and silver nanoparticles for multifunctional conductive biopolymer composites," Carbon, vol. 49, no. 7, pp. 2370-2379, 2011.

[2] G. D. Liang, S. P. Bao, and S. C. Tjong, "Microstructure and properties of polypropylene composites filled with silver and carbon nanotube nanoparticles prepared by melt-compounding," Materials Science and Engineering B, vol. 142, no. 2-3, pp. 55-61, 2007.

[3] P. C. Ma, B. Z. Tang, and J.-K. Kim, "Effect of CNT decoration with silver nanoparticles on electrical conductivity of CNTpolymer composites," Carbon, vol. 46, no. 11, pp. 1497-1505, 2008.

[4] J. Zhao, A. Buldum, J. Han, and J. P. Lu, "Gas molecule adsorption in carbon nanotubes and nanotube bundles," Nanotechnology, vol. 13, no. 2, pp. 195-200, 2002.

[5] C. N. R. Rao, U. Maitra, K. S. Subrahmanyam et al., "Potential of nanocarbons and related substances as adsorbents and chemical storage materials for $\mathrm{H}_{2}, \mathrm{CO}_{2}$ - and other gases," Indian Journal of Chemistry A, vol. 51, no. 1-2, pp. 15-31, 2012.

[6] K. Y. Dong, J. Choi, Y. D. Lee et al., "Detection of a $\mathrm{CO}$ and $\mathrm{NH}_{3}$ gas mixture using carboxylic acid-functionalized single-walled carbon nanotubes," Nanoscale Research Letters, vol. 8, article 12, 2013.

[7] F. Xu, M. Barberio, R. Vasta, A. Bonanno, and V. Pirronello, "Vacuum system," Physical Review Special Topics, vol. 11, no. 11, Article ID 113201, 2008.

[8] M. Barberio, P. Barone, V. Pingitore, A. Bonanno, and F. Xu, "Photoluminescence from silver/carbon nanotubes composites," Superlatice and Microstructures, vol. 57, pp. 129-138, 2013.

[9] K. L. Kelly, E. Coronado, L. L. Zhao, and G. C. Schatz, "The optical properties of metal nanoparticles: the influence of size, shape, and dielectric environment ", The Journal of Physical Chemistry B, vol. 107, no. 3, pp. 668-677, 2003.

[10] M. Barberio, P. Barone, V. Pingitore, and A. Bonanno, "Optical properties of $\mathrm{TiO}_{2}$ anatase: carbon nanotubes composites studied by cathodoluminescence spectroscopy," Superlattices and Microstructures, vol. 51, no. 1, pp. 177-183, 2012.

[11] Y. Yao, G. Li, S. Ciston, R. M. Lueptow, and K. A. Gray, "Photoreactive $\mathrm{TiO}_{2}$ /carbon nanotube composites: synthesis and reactivity," Environmental Science and Technology, vol. 42, no. 13, pp. 4952-4957, 2008.

[12] M. Barberio, R. Vasta, P. Barone, G. Manicò, and F. Xu, "Experimental and theoretical study on the ethane and acetylene formation from electron irradiation of methane ices," World Journal of Condensed Matter Physics, vol. 3, no. 1, pp. 14-20, 2013.

[13] M. Barberio, P. Barone, R. Vasta, G. Manicò, and F. Xu, "Formation of molecular nitrogen and diazene by electron irradiation of solid ammonia," Thin Solid Films, vol. 520, no. 16, pp. 54795481, 2012.
[14] NIST XPS data base, http://srdata.nist.gov/xps/main_search_ menu.aspx.

[15] P. A. Redhead, “Thermal desorption of gases," Vacuum, vol. 12, no. 4, pp. 203-211, 1962. 

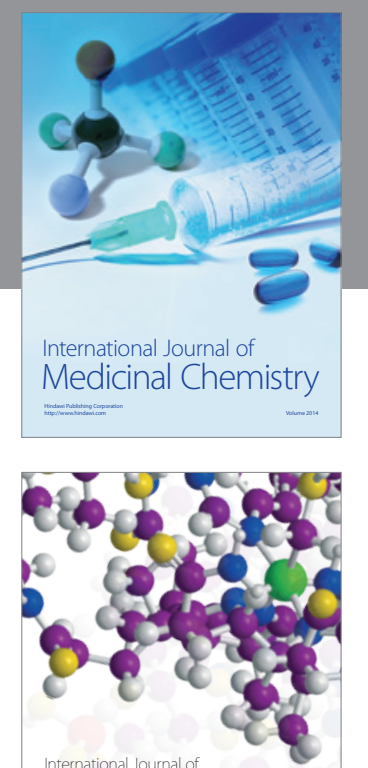

\section{Carbohydrate} Chemistry

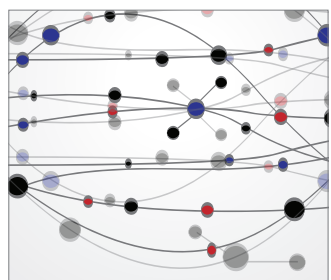

The Scientific World Journal
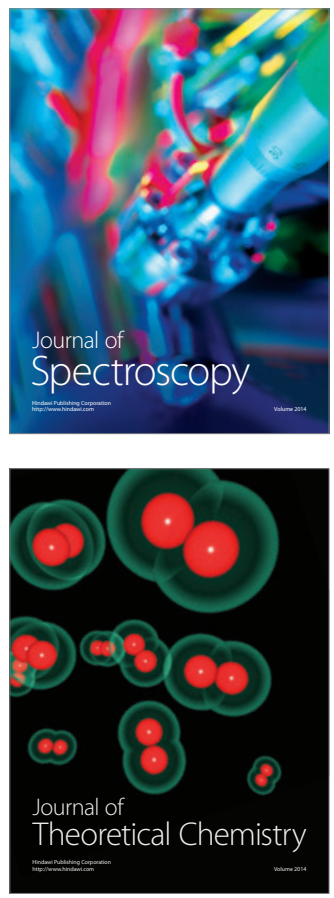
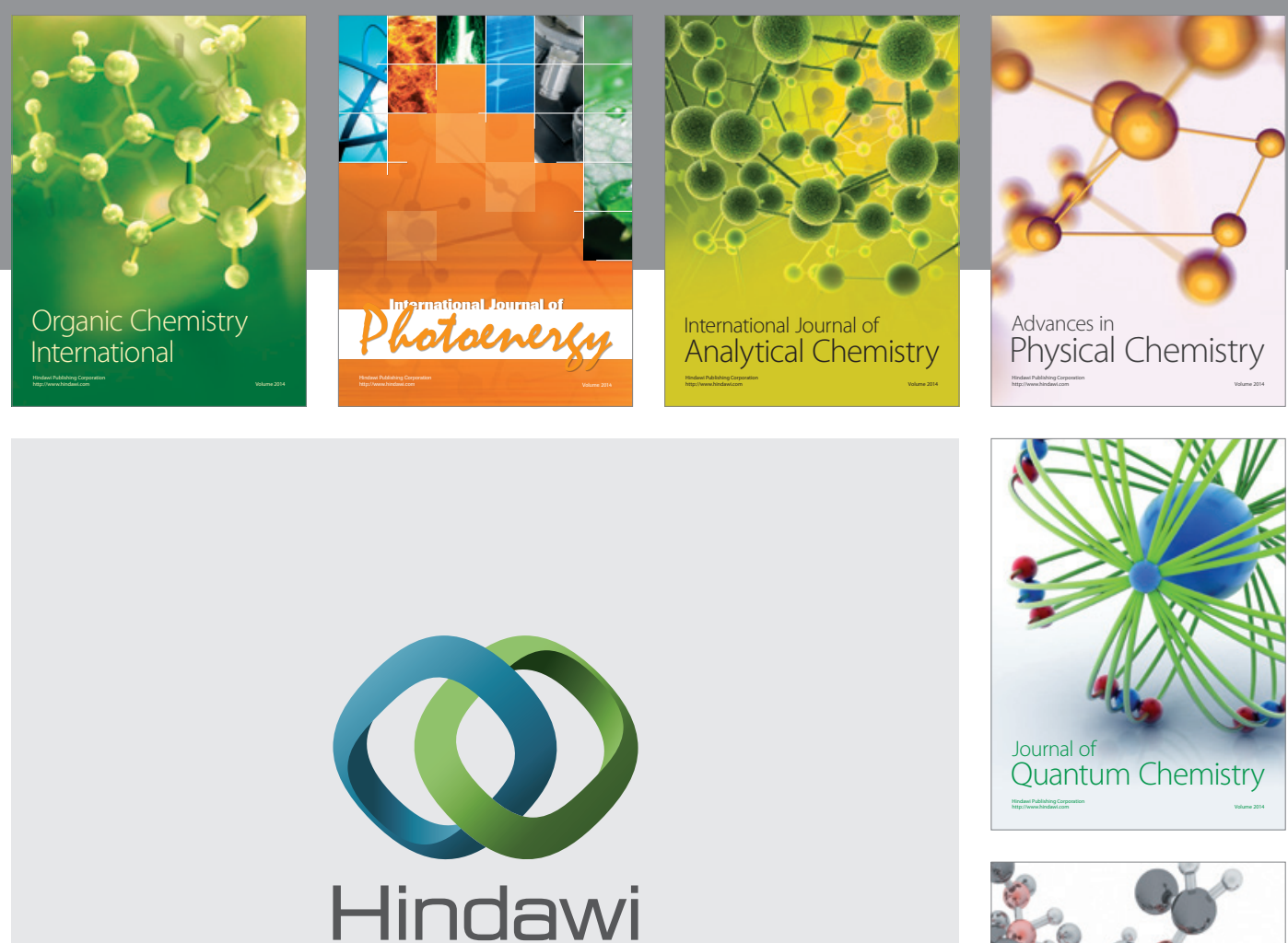

Submit your manuscripts at

http://www.hindawi.com

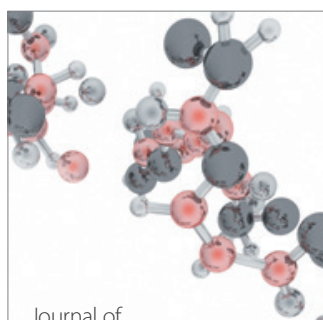

Analytical Methods

in Chemistry

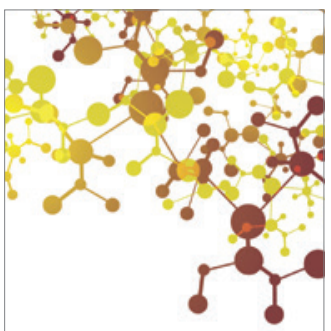

Journal of

Applied Chemistry

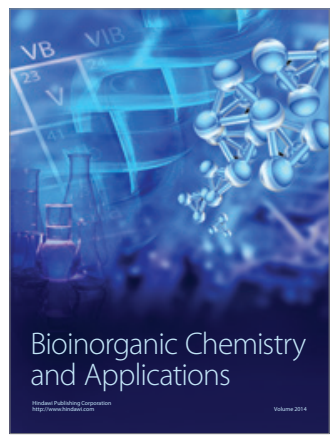

Inorganic Chemistry
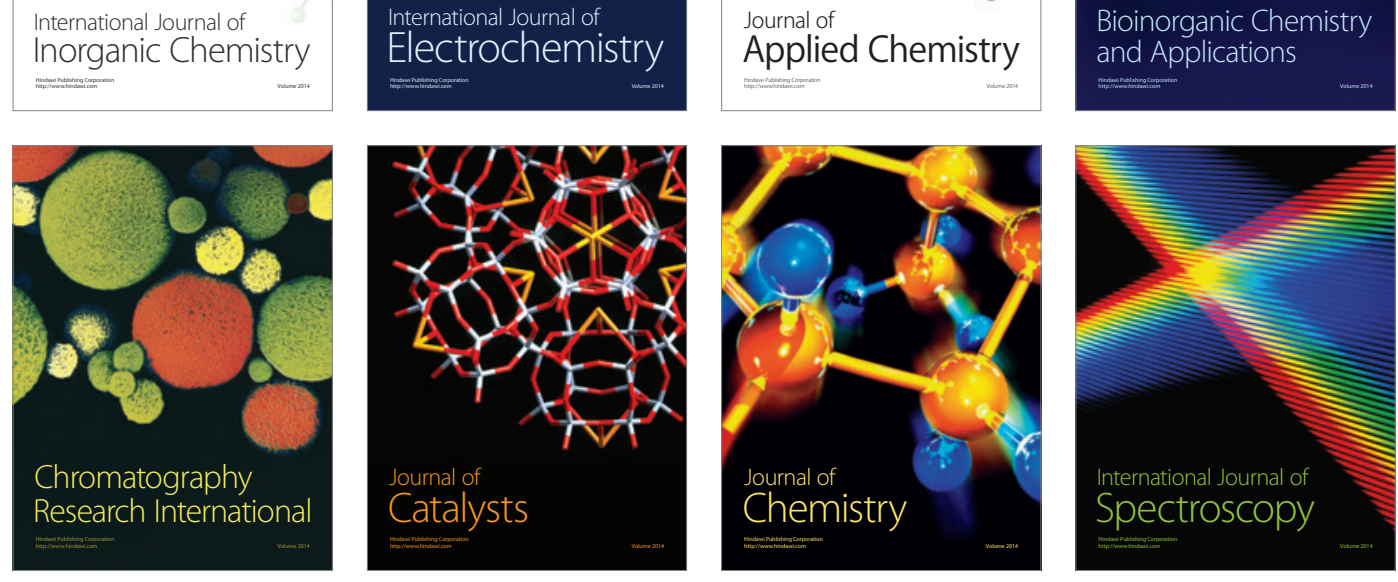